\title{
The Effects of Cracks on Calorimeter Response in the SDC "Dogleg" Design
}

\author{
Dan Green \\ Fermi National Accelerator Laboratory \\ P.O. Box 500, Batavia, Illinois 60510
}

\section{DISCLAIMER}

\begin{abstract}
This report was prepared as a
Government. Neither the United States of work sponsored by an agency of the United States employees, makes any warranty, express Government nor any agency thereof, nor any of their bility for the accuracy, completeness, or usefulness, or assumes any legal liability or responsiprocess disclosed, or represents that its use wulness of any information, apparatus, product, or ence herein to any specific commercial product, process infringe privately owned rights. Refermanufacturer, or otherwise does not necessarily const, or service by trade name, trademark, mendation, or favoring by the United States Governmte or imply its endorsement, recomand opinions of authors expressed herein do not necest or any agency thereof. The views United States Government or any agency thereof.
\end{abstract}

November 1992

\section{MASTER}




\section{Disclaimer}

This report was prepared as an account of work sponsored by an agency of the United States Government. Neither the United States Government nor any agency thereof, nor any of their employees, makes any warranty, express or implied, or assumes any legal liability or responsibility for the accuracy, completeness, or usefulness of any information, apparatus, product, or process disclosed, or represents that its use would not infringe privately owned rights. Reference herein to any specific commercial product, process, or service by trade name, trademark, manufacturer, or otherwise, does not necessarily constitute or imply its endorsement, recommendation, or favoring by the United States Government or any agency thereof. The views and opinions of authors expressed herein do not necessarily state or reflect those of the United States Government or any agency thereof. 


\title{
THE EFFECTS OF CRACKS ON CALORIMETER
}

\author{
RESPONSE IN THE SDC "DOGLEG" DESIGN
}

\author{
Dan Green \\ Fermi National Accelerator Laboratory \\ Batavia, Ilinois
}

\section{1. "Complete Cracks"}

In an initial attempt to set the scale for cracks, the reaction $\mathrm{H}(800)$ $\rightarrow Z Z \rightarrow(q+q)+(\tau 1+\tau 2) \rightarrow(\rho 1+v 1)+(\rho 2+v 2)$ was studied. The figure of merit was taken to be the $Z$ transverse mass, i.e. the mass of $\left(\rho 1+2+\mathbf{E}_{T}\right)$. With no cracks in the coverage save the beam holes at $\eta=5$, this quantity has a fractional spread of $\sim 12 \%$. The additional contribution of cracks was unfolded in quadrature. The results as a function of the width of the crack at the EM surface are shown in Fig. 1.

Clearly, the scale where the effects of cracks becomes important for this physics process is a few $\mathrm{cm}$ (few $\%$ of azimuth). At a full crack width of $4 \mathrm{~cm}$, the additional contribution due to cracks is comparable to all the other effects intrinsic to the decay kinematics and to the SDC baseline detector. Note that these cracks are "complete", in that it is assumed that all energy incident on them is completely lost. Since the barrel wedge in SDC will have an irreducible dead space of width, $\sim 1 \mathrm{~cm}$ due to fiber routing, light tightening, etc., it was decided to study a "dogleg" geometry.

\section{The Dogleg Geometry}

The geometry which was chosen for study is shown in Fig. 2. There is a "projective" crack in the first two compartments (EM and HAD1), followed by a "dogleg" prior to the HAD2 compartment. There are two types of barrel wedge. The final one used in assembly is the "keystone" of the barrel arch. This arrangement is charge symmetric, and always has $>5$ absorption lengths of protection against energy leakage. Other possibilites, e.g. "bicycle spoke", are not charge symmetric or, e.g. purely projective cracks have more leakage. The dogleg geometry is mechanically more difficult, but does not suffer from these defects. 


\section{Jets as Probes of Cracks}

It is likely that the highest momentum objects incident on the calorimeter will be jets. Dijet production is a rate limited process at the SSC. The highest available PtJ in 1 SSC year is about $5 \mathrm{TeV}$ [1]. A simple model of jet fragmentation was constructed, with a longitudinal fragmentation function $D(z)=(1-z)^{6} / z$, where $k=z P t J$. This function is shown, as generated, in Fig. 3. The $1 / z$ behavior of $D(z)$ at low $z$ is evident. The transverse momentum fragmentation shape is shown in Fig. 4. The typical momentum transverse to the jet axis is, $\langle\mathrm{kt}\rangle \sim 1 \mathrm{GeV}$. Finally, the jet multiplicity is shown in Fig. 5. The procedure is to pick particles with $z$ out of $D(z)$ until the sum of $z$ is $=1$. This method leads to a mean multiplicity which increases logarithmically with $\mathrm{PtJ},\langle n\rangle \sim \ln (\mathrm{PtJ})$. At 5 $\mathrm{TeV}$, the mean multiplicity is $\sim 70$.

\section{Model for Photon and Hadron Showers}

In the interest of simplicity, showers were represented by parameterized shapes. The EM showers are represented as in the Particle Data Group Report [2]. The energy deposited in any finite length calorimeter is represented by the incomplete ganima function, as shown in Fig. 6. The fluctuations in EM showers are assumed to be limited entirely to fluctuations in the conversion point.

For hadron showers, the two component model given by UA1, [3], is assumed. The form used is also shown in Fig. 6. There is an EM component of "strength" fo, and an hadronic component, which carries an energy fraction (1-fo). Shower fluctuations are assumed to be represented by interaction point fluctuations, and by shower to shower differences in the neutral content of the first interaction, which was chosen to be Gaussian with a mean of $\langle f o\rangle=0.46$ and a standard deviation of, dfo $=0.2$.

The SDC calorimeter is assumed to consist of, at 90 degrees, an EM compartment of 20 Xo depth, a HAD1 compartment of 4.2 interaction lengths, and a HAD2 (coarse hadronic) compartment of depth, $\mathrm{D}=5$ (measured in interaction lengths).

The response of this simplified model to a $1 \mathrm{TeV}$ electron is shown in Fig. 7. The $e$ is largely contained in the EM compartment. The residual leakage is contained in the HADl compartment. For an incident $1 \mathrm{TeV}$ hadron, the energy deposition for 100 showers is shown in Fig 8. Most of the energy is contained in HAD1. Few hadrons convert and deposit energy in EM. HAD2 catches the leakage energy. The small amount of lost energy, EL, is less than $2 \%$ of the incident energy.

Single photons and hadrons are then shot into the dogleg, incident on $\mathrm{HAD} 2$ alone. For $1 \mathrm{TeV}$ e, corresponding to the "leading" fragment of a $5 \mathrm{TeV}$ jet, the shower is almost completely contained. Note that HAD2 is $\sim 50$ Xo deep. Therefore, the containment seen in Fig. 9, is better than the EM compartment containment shown previously in Fig. 7. In Fig. 10, the leakage for an incident hadron is shown. The leakage energy fraction for an incident hadron is substantial [4]. The mean energy fraction lost is $\sim 20 \%$. Since this situation corresponds to the "leading" fragment of a $5 \mathrm{TeV}$ jet incident on the "dogleg" crack, we may expect substantial jet leakage in this case also. 


\section{5. $5 \mathrm{TeV}$ Jets and SDC Hermeticity}

A $5 \mathrm{TeV}$ jet, fragmented into an ensemble of about 70 neutral and charged pions, was incident on the SDC "toy model" calorimeter. The resulting calorimetric energy distributions for the compartments and the leakage for 100 jets with $\mathrm{PtJ}=5 \mathrm{TeV}$ are shown in Fig. 11. Roughly 35\% of the energy appears in EM (photons and early pion interactions), $50 \%$ appears in HAD1. Only $15 \%$ appears in HAD2, and $<2 \%$ leaks out the back. In fact, jets of this type were studied in choosing the depth of SDC calorimetry [4].

For the dogleg, an azimuthal crack was assumed, as shown in Fig. 2. The full width of the crack between EM and HAD1 for adjacent wedges was assumed to be $2 \mathrm{~cm}$. The crack was assumed to be of infinite extent, which means the probiem is essentially 2 dimensional. The shower transverse size was not considered. Incident fragments which enter the crack see a reduced calorimeter depth. The minimal depth for any fragment is the total depth of HAD2. To set the scale, a fragment has $\langle\mathrm{k}\rangle=1 \mathrm{GeV}$. If the jet axis is exactly incident on the center line of the crack, then any fragment with an angle $\sim 1 \mathrm{~cm} / \mathrm{R}=$ $200 \mathrm{~cm}$, (5 mrad) with respect to the jet axis, will enter the crack. For $\langle\mathrm{kt}\rangle$, this means any fragment with $\mathrm{k}>200 \mathrm{GeV}$. Therefore, most of the jet energy will see a depth of only $\mathrm{D}=5$, in the case that the jet axis is along the crack center line.

The distribution of energies of 100 jets incident exactly on the center of the crack are shown in Fig 12. Note that the EM energy is essentially zero. The HAD1 energy is also much reduced with respect to the distribution shown in Fig. 11. In fact, most of the jet energy is carried by the leading fragments. For the assumed $\mathrm{D}(\mathrm{z})$, the first fragment has, on average, $\langle z 1\rangle=0.23$. Clearly, HAD2 absorbs the majority of the energy. The leakage energy is also much increased. The fractional ieakage energy is $\sim 10 \%$. Therefore, for the highest energy jets, the crack must be excluded from the "fiducial" region. A similar study of $400 \mathrm{GeV}$ jets, [5], is in rough agreement with this conclusion.

\section{Fiducial Regions}

Given the enhanced leakage near the crack, the question of the size of the fiducial cuts naturally arises. An ensemble of 100 jets with $5 \mathrm{TeV}$ energy was swept across the crack. The means of the EEM, EHAD1, EHAD2, and EL distributions are shown in Fig. 13. Clearly, jets incident $>1 \mathrm{~cm}$ away from the edge boundary of the crack are well contained. Therefore, Fig. 13 serves as an interpolation between Fig. 11 and Fig. 12. It is expected that jets of lesser energies will be better contained [5]. 


\section{References}

1. Solenoidal Detector Collaboration Technical Design Report, April 1, 1992, SDC-92201.

2. Review of Particle Properties Phys. Rev. D 4511 (1992).

3. Bock et al. Nucl. Inst. \& Meth. 186, 533 (1981).

4. D. Green et al. Depth Requirements in SSC Calorimeters, Fermilab, FN-570 (1991).

5. C. Hearty, Private Communication. 


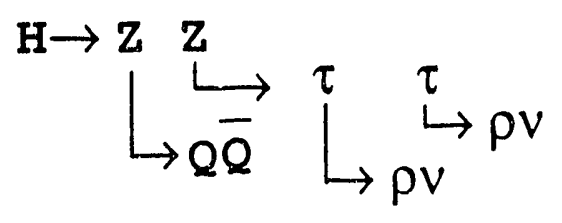

$$
\mathbf{M}_{T Z}=\mathbf{M}_{T}\left(\rho \rho E_{T}\right)
$$

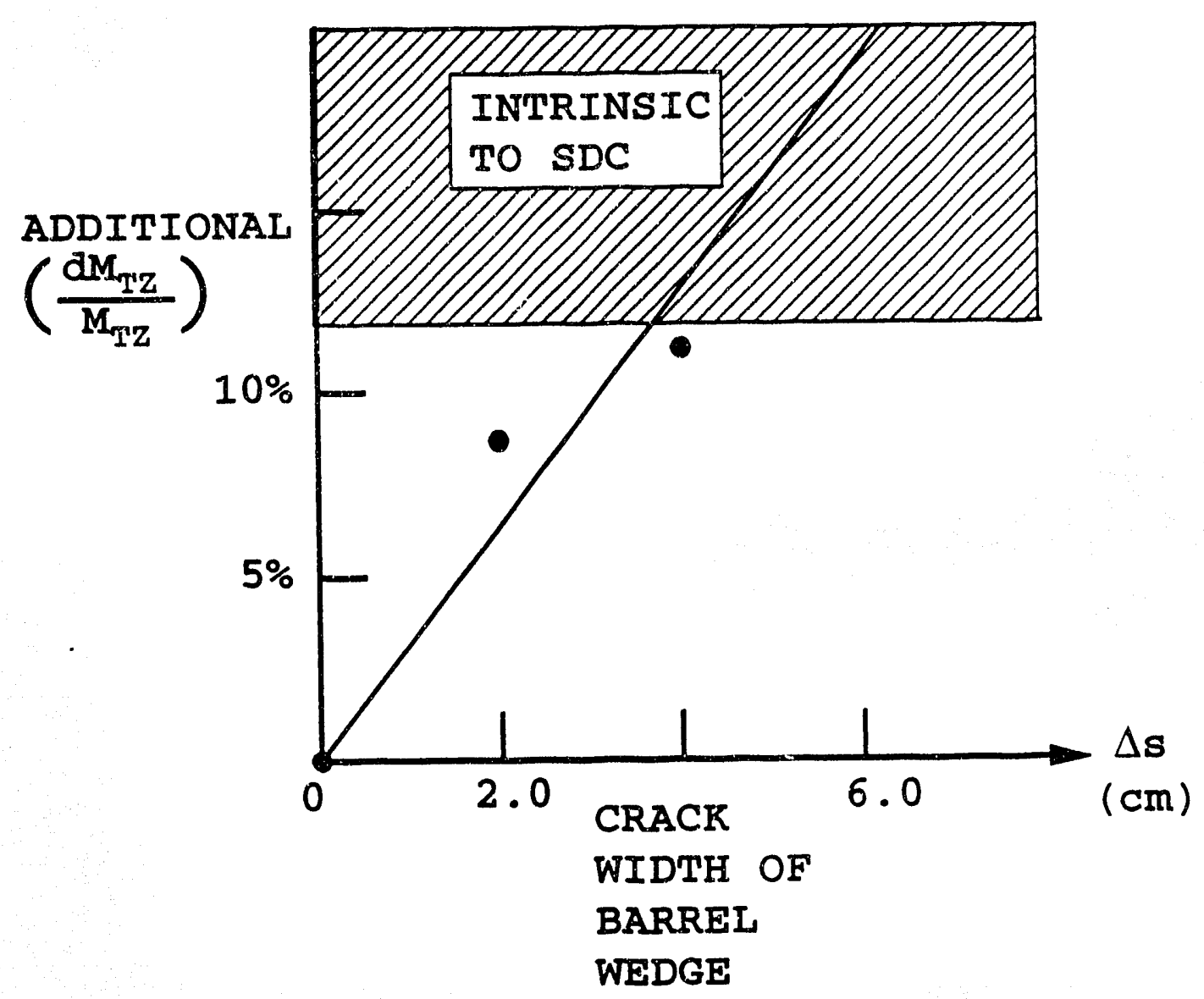

1. Induced fractional tranverse mass error for the $\mathrm{Z}$ boson as a function of crack width. The "intrinsic" error due to all other effects in SDC is also indicated. The physics reaction is $\mathrm{H}(800) \rightarrow Z+Z, Z \rightarrow q q, Z \rightarrow \tau+\tau, \tau \rightarrow \rho+v$. 

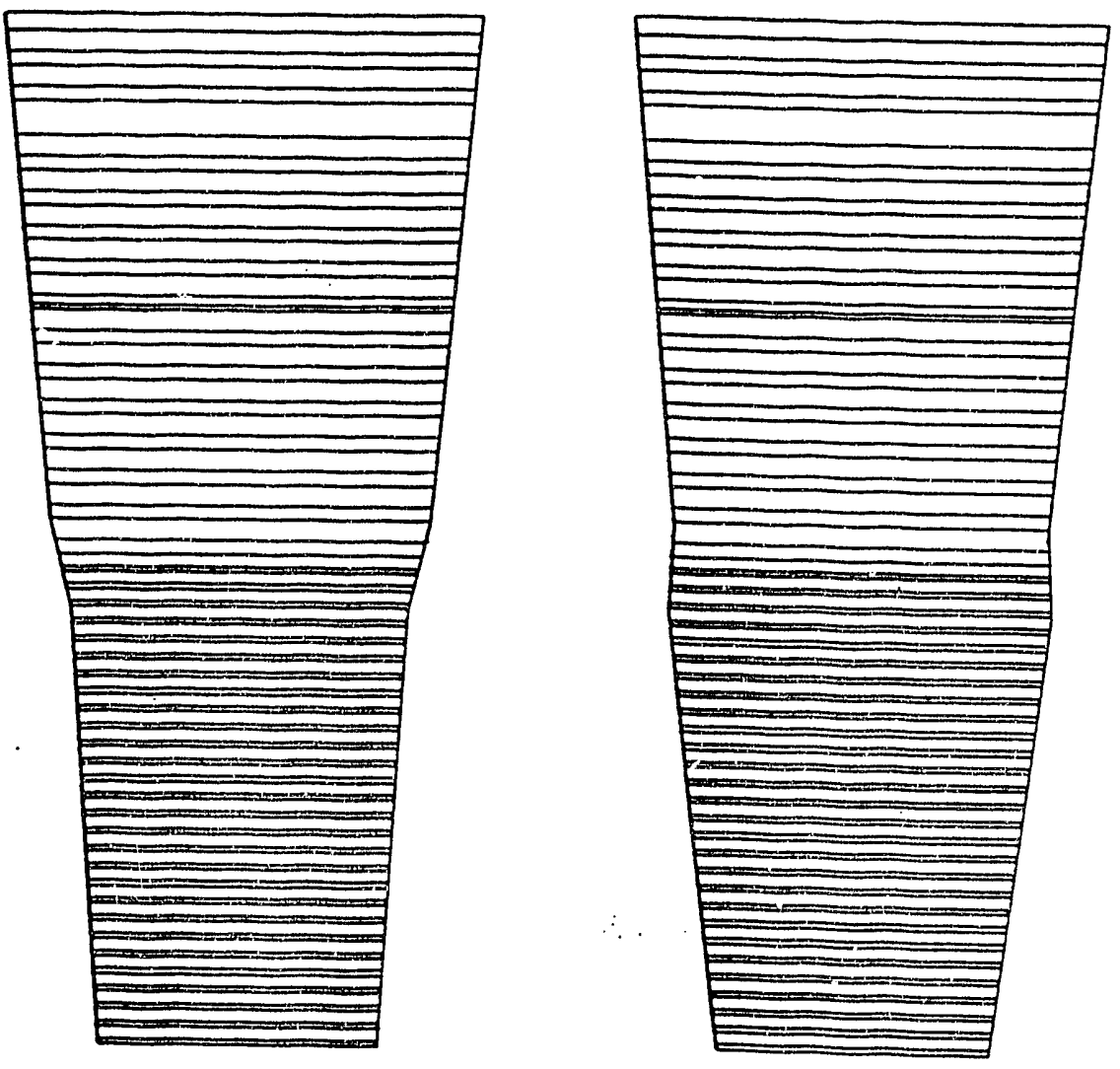

2. SDC barrel calorimeter with "dogleg" construction. There are 2 types of "wedge" modules which differ in the azimuthl extent of the second hadronic compartment (HAD2). 


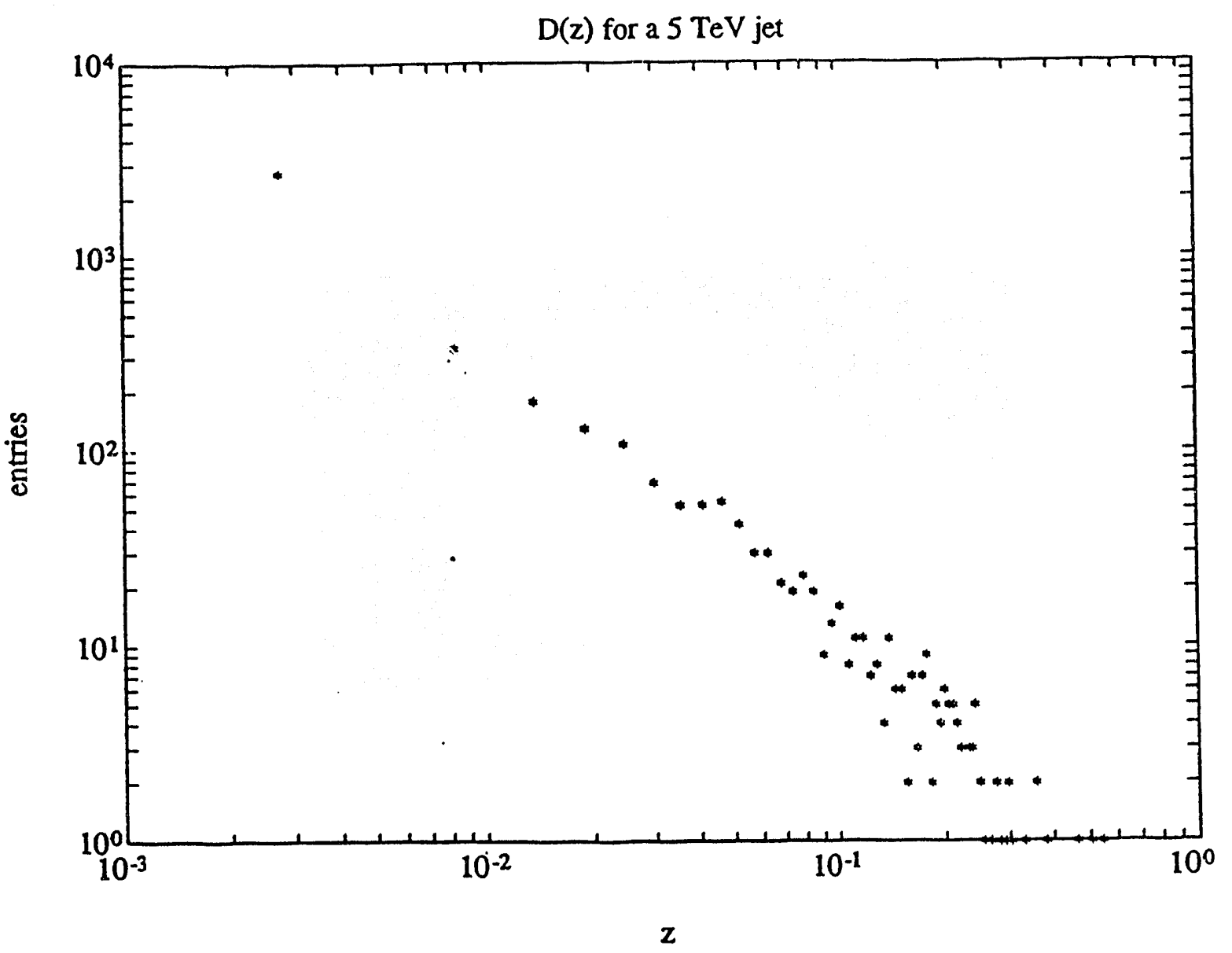

3. Longitudinal fragmentation function, $D(z)$, assumed for jets. The form used was $(1-z)^{6} / z$ with $z>m_{\pi} / \mathrm{Pj}$. 


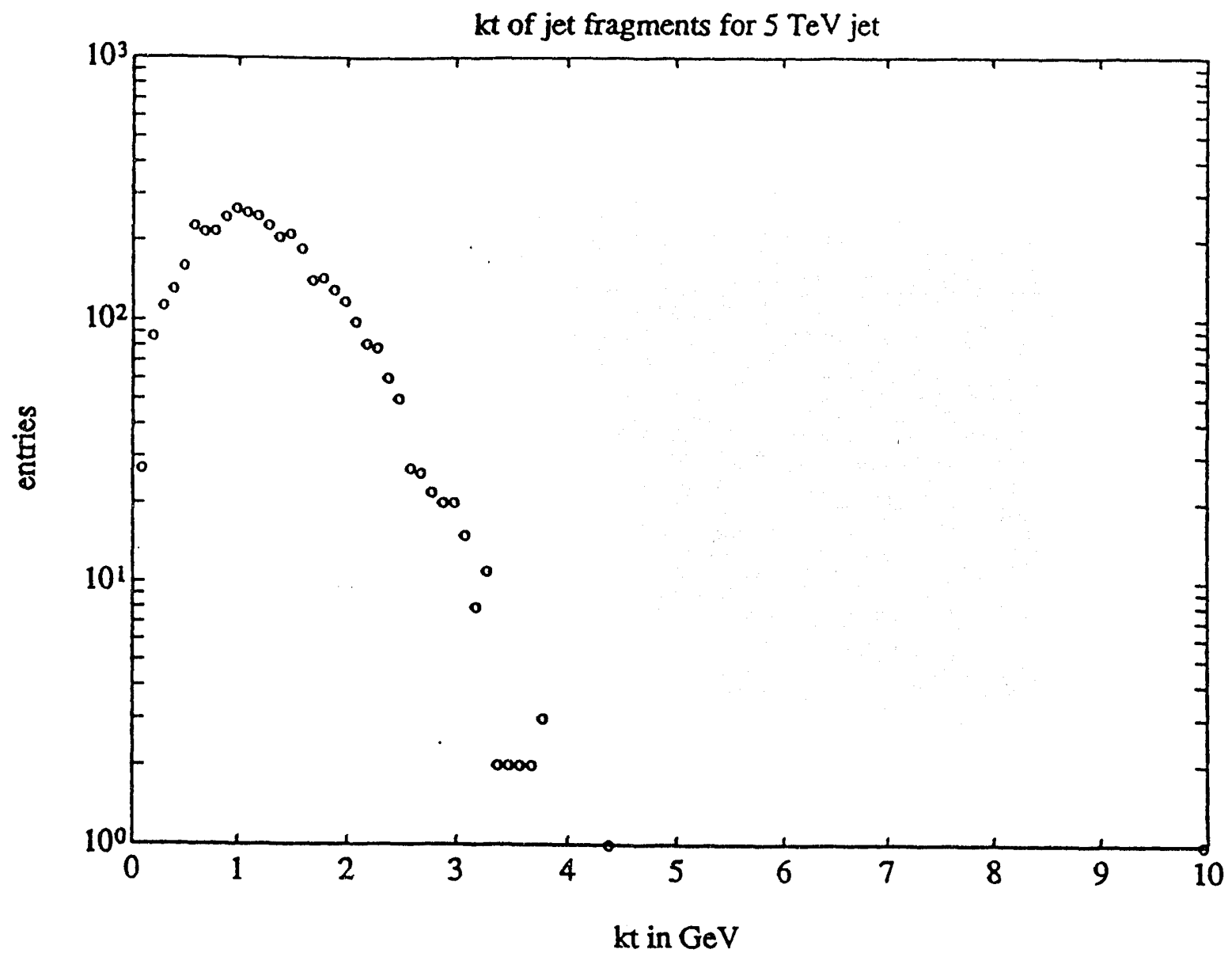

4. Transverse fragmentation function. The assumed form is exponential in $k_{t}^{2}$. The mean transverse momentum is $\langle\mathrm{kt}\rangle \sim 1 \mathrm{GeV}$. 


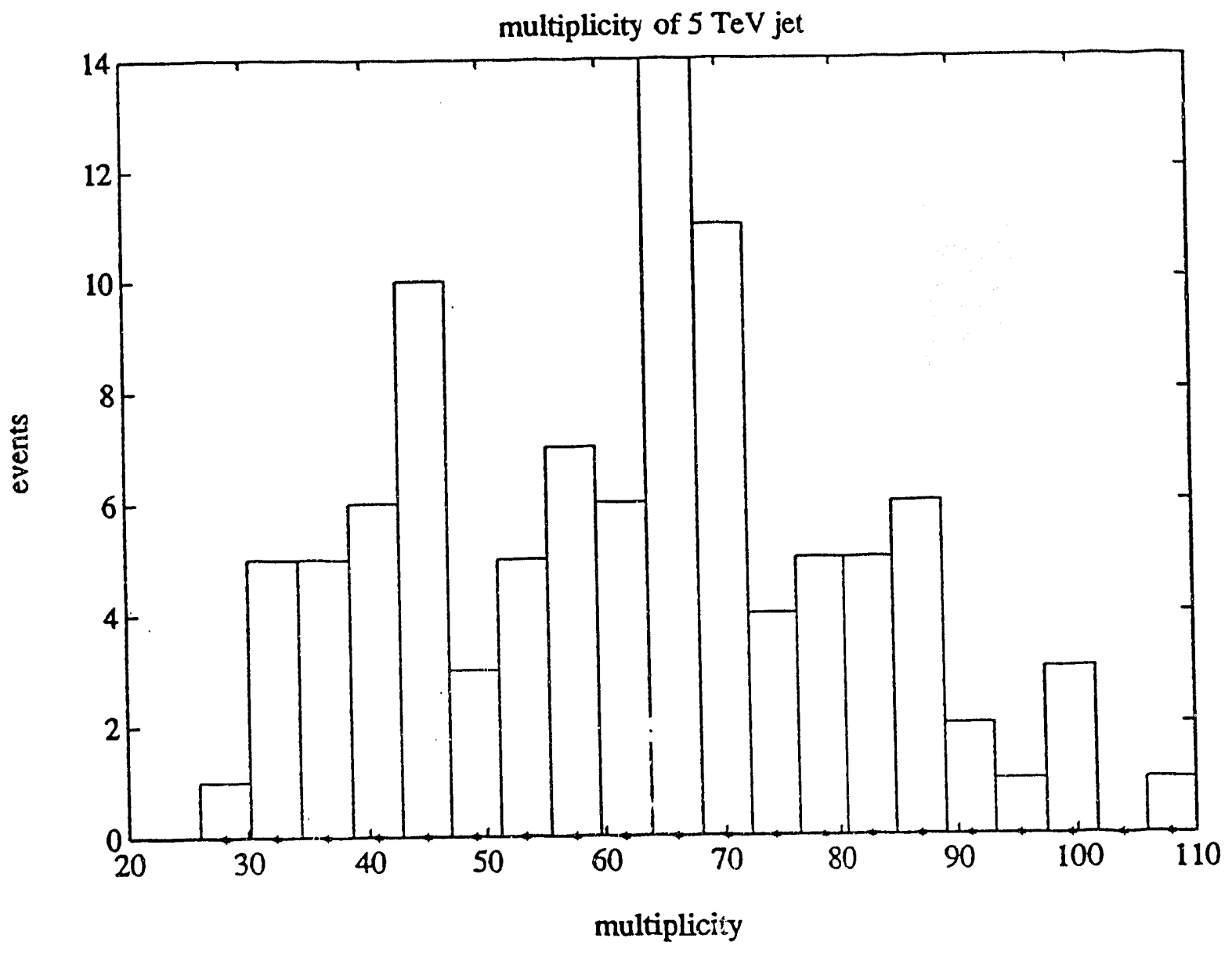

5. Multiplicity of jet fragments for a $5 \mathrm{TeV}$ jet. The mean jet multiplicity grows logarithmically with $\mathrm{Pj}$. For a $5 \mathrm{TeV}$ jet, $\langle\mathrm{n}\rangle \sim 70$. 


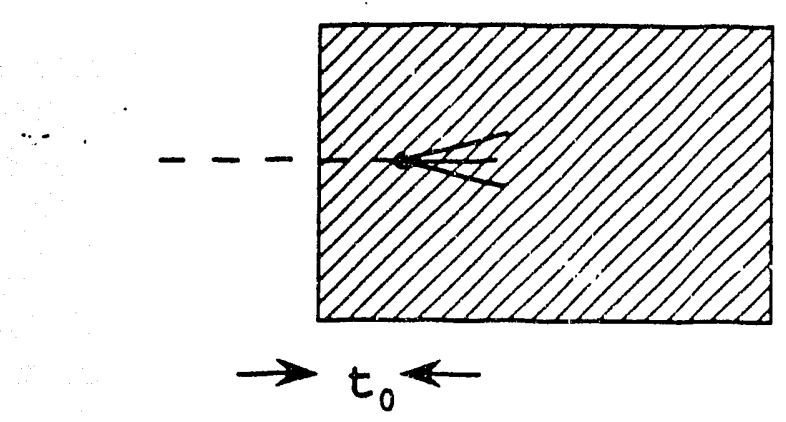

$$
\begin{aligned}
\frac{d E}{E_{0}} & =u^{a-1} e^{-u} d u / \Gamma(a) \\
u & =b t \quad t=z / x_{0}
\end{aligned}
$$

$\mathrm{E} / \mathrm{E}_{0}=\gamma\left(a, \mathbf{u}_{0}\right) / \Gamma(a)$

$$
\frac{d E}{E_{0}}=\left\{f_{0}\left[u^{a-1} e^{-u} d u\right]+\left(1-f_{0}\right)\left[v^{a-1} e^{-v} d v\right]\right\} / \Gamma(a)
$$

$E / E_{0}=\left\{f_{0}\left[\gamma\left(a, u_{0}\right)\right]+\left(1-f_{0}\right)\left[\gamma\left(a, v_{0}\right)\right]\right\} / \Gamma(a)$

$$
\begin{aligned}
D & =z / \lambda_{0} \\
v & =d D \\
d f_{0} & \sim 0.2
\end{aligned}
$$

6. Assumed shower shapes for photons and hadrons. For photons the shower shape, at fixed energy $E$, is fixed. All fluctuations come from conversion point variations. Depths are measured in $t$, scaled to Xo units. For hadrons the shower composition of neutrals/charged is fluctuated, $\left\langle f_{0}\right\rangle=0.46, \mathrm{dfo}=0.2$. The conversion point also varies. Depth is measured in Xo and interaction length units, D, for the EM and hadronic components separately. 

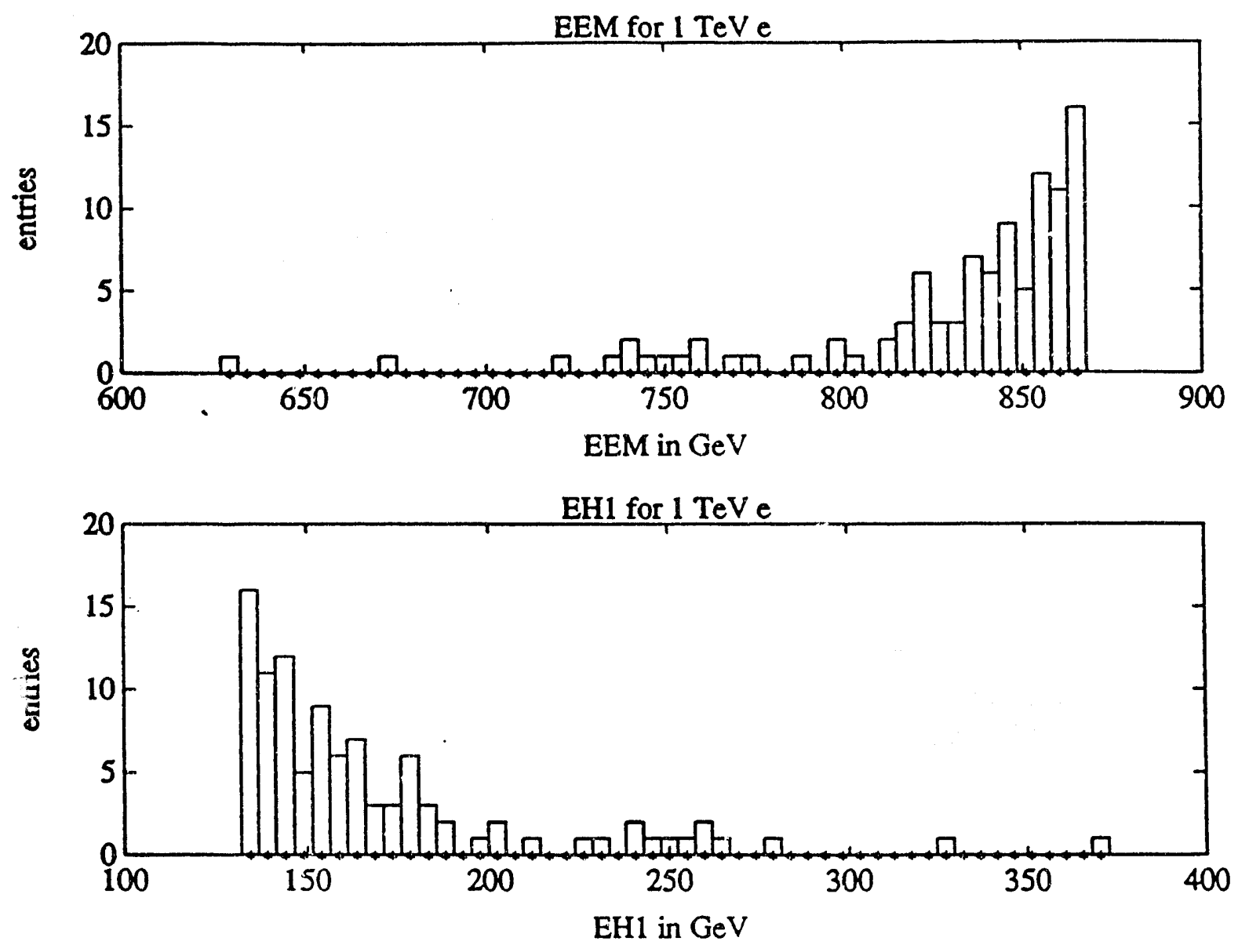

7. Deposition of energy in EM and HADl compartments for a $1 \mathrm{TeV}$ e. That energy roughly corresponds to the leading fragment of a $5 \mathrm{TeV}$ jet. 

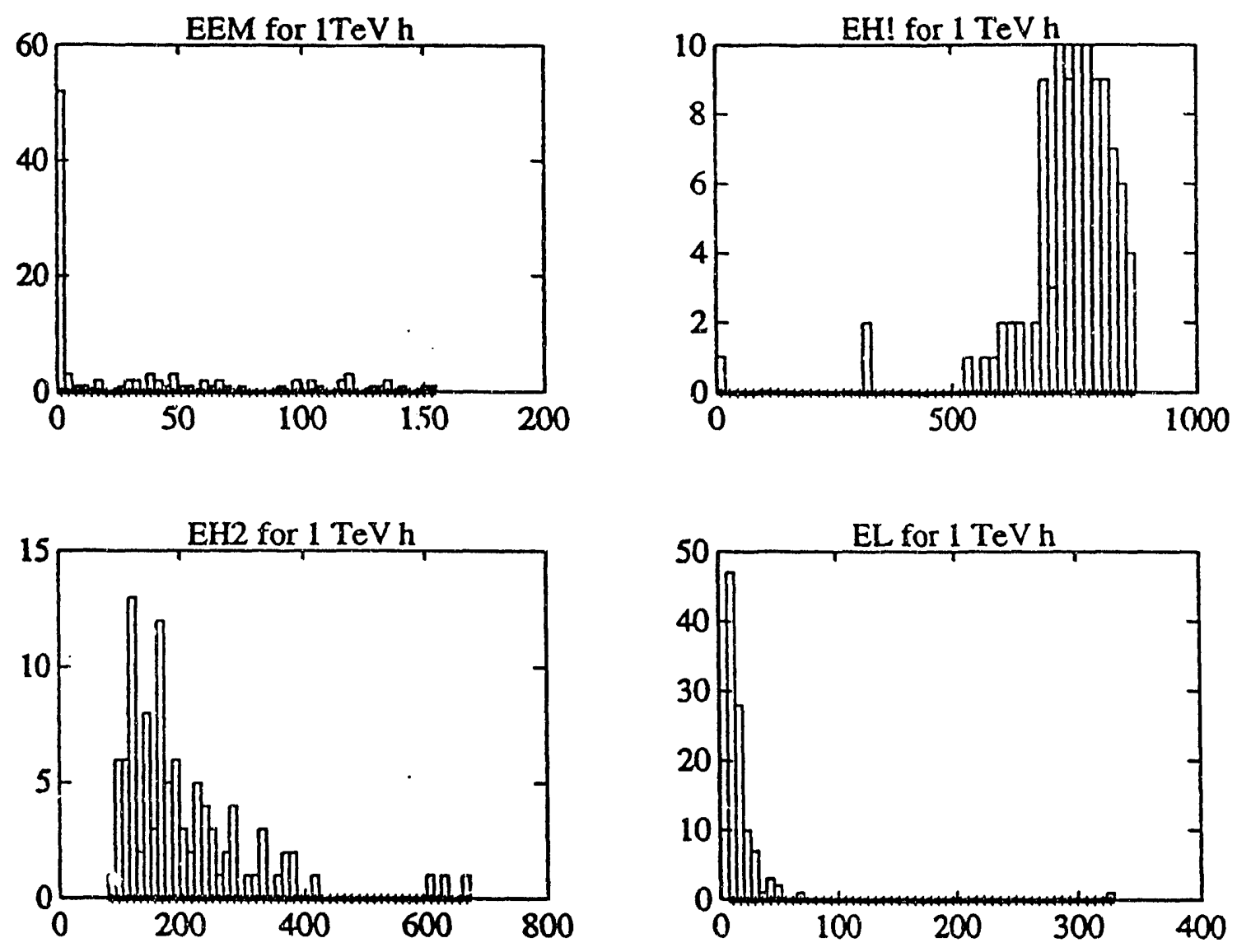

8. Deposition of energy in EM, HAD1, and HAD2 compartments for a $1 \mathrm{TeV}$ hadron.

The leakage energy, EL, is also shown for completeness. 

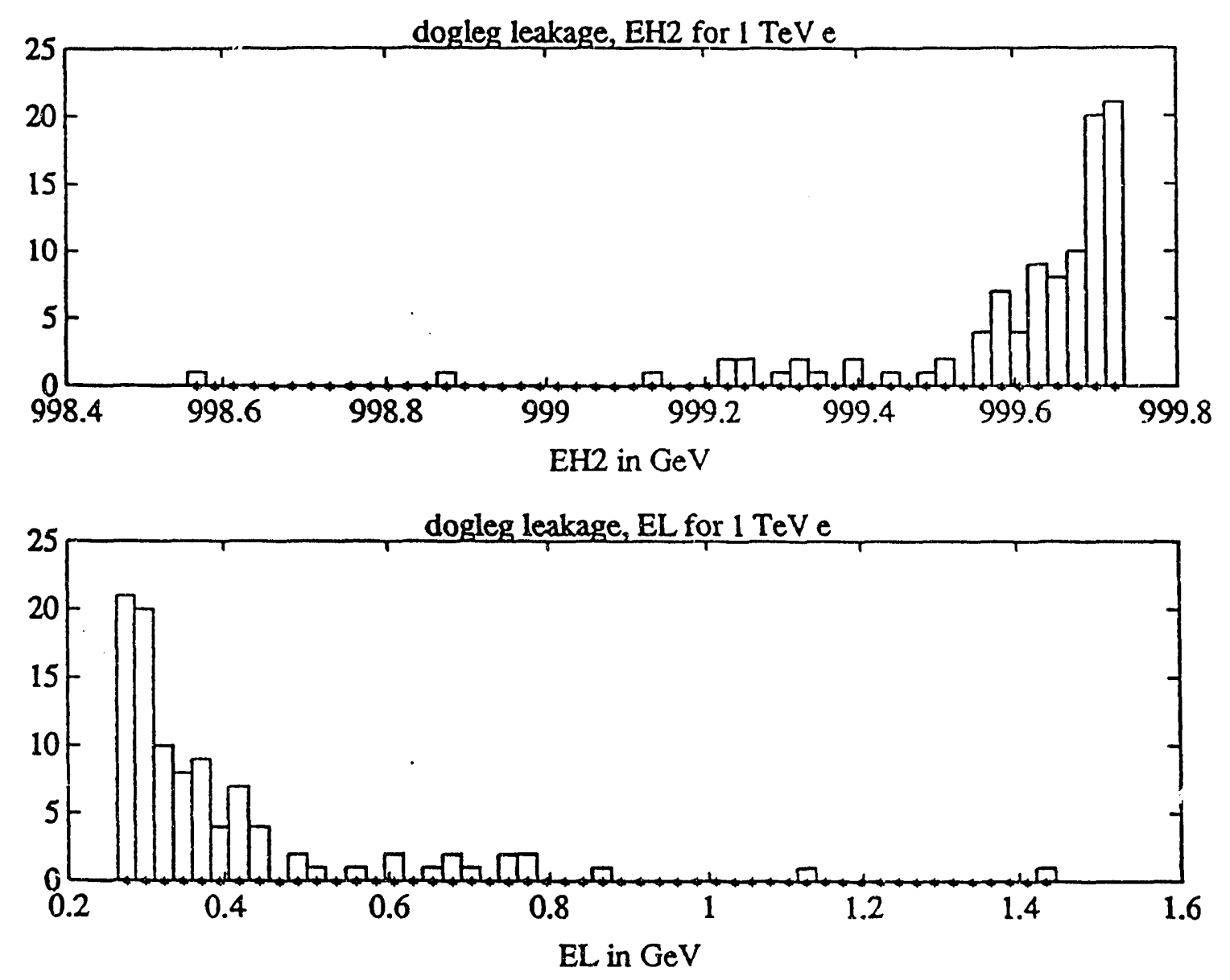

9. Energy deposition in HAD2 and EL for a $1 \mathrm{TeV}$ photon incident on the HAD2 dogleg. This situation corresponds to the leading $\mathrm{f}_{\mathrm{t}}$ sment of a $5 \mathrm{TeV}$ jet being neutral and passing EM and HAD1 and striking the HAD2 "dogleg". 

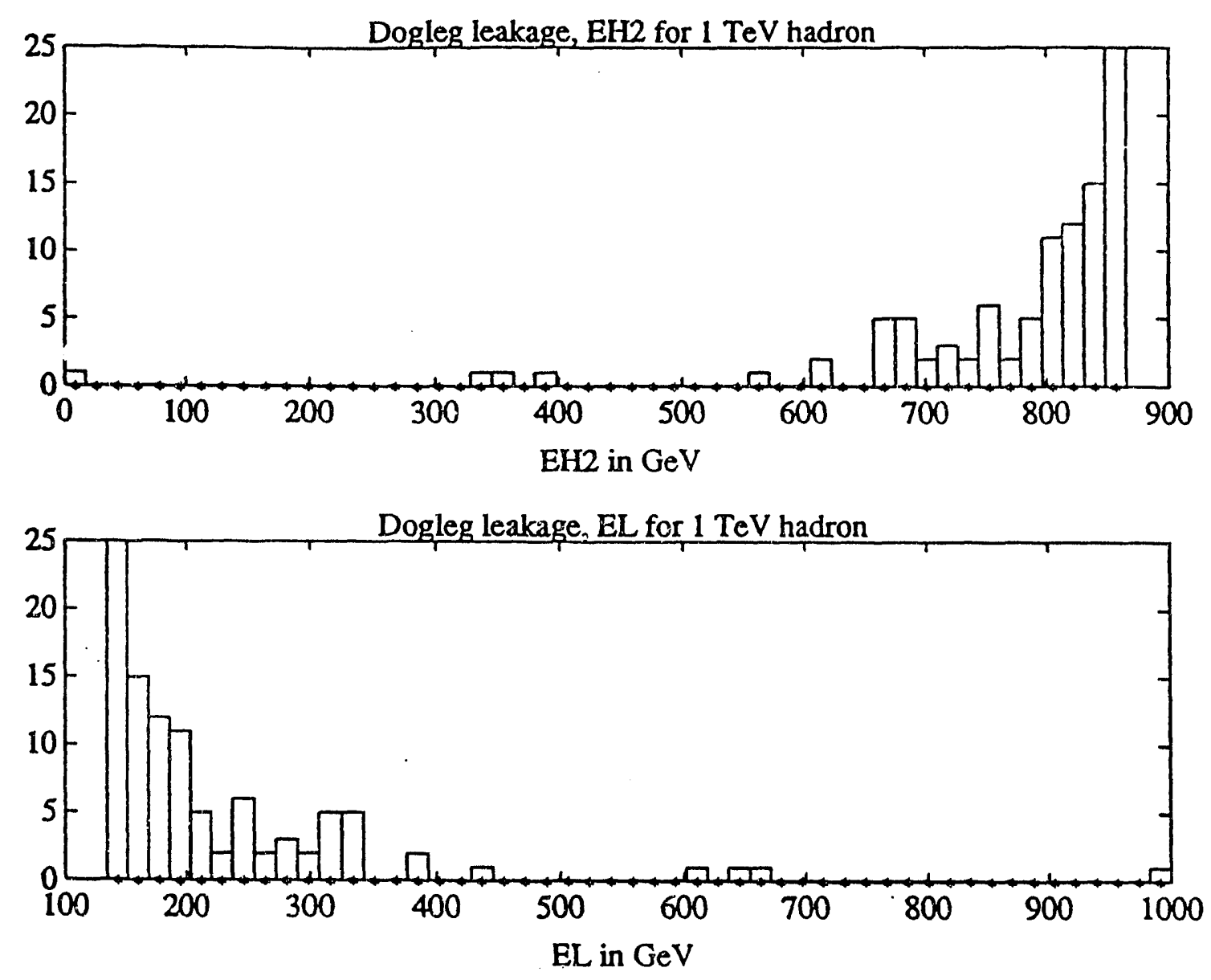

10. Energy deposition in HAD2 and EL for a $1 \mathrm{TeV}$ hadron incident on the HAD2 dogleg. This situation corresponds to the leading fragment of a $5 \mathrm{TeV}$ jet being charged, passing EM and HAD1 in the crack, and striking the HAD2 "dogleg". 

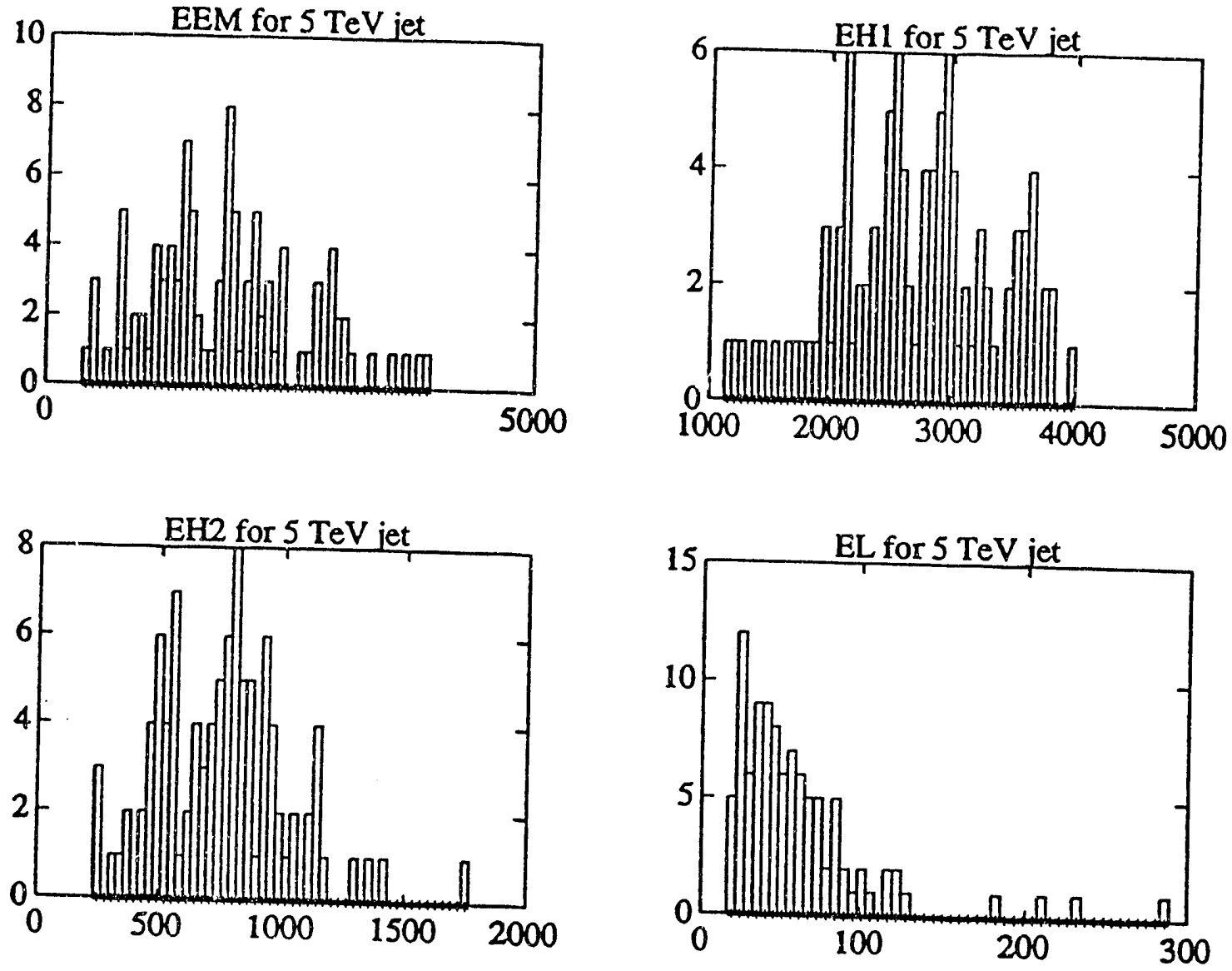

11. Energy deposition in EM, HAD1, HAD2, and EL for a $5 \mathrm{TeV}$ jet incident on the SDC calorimeter far from the "crack". 

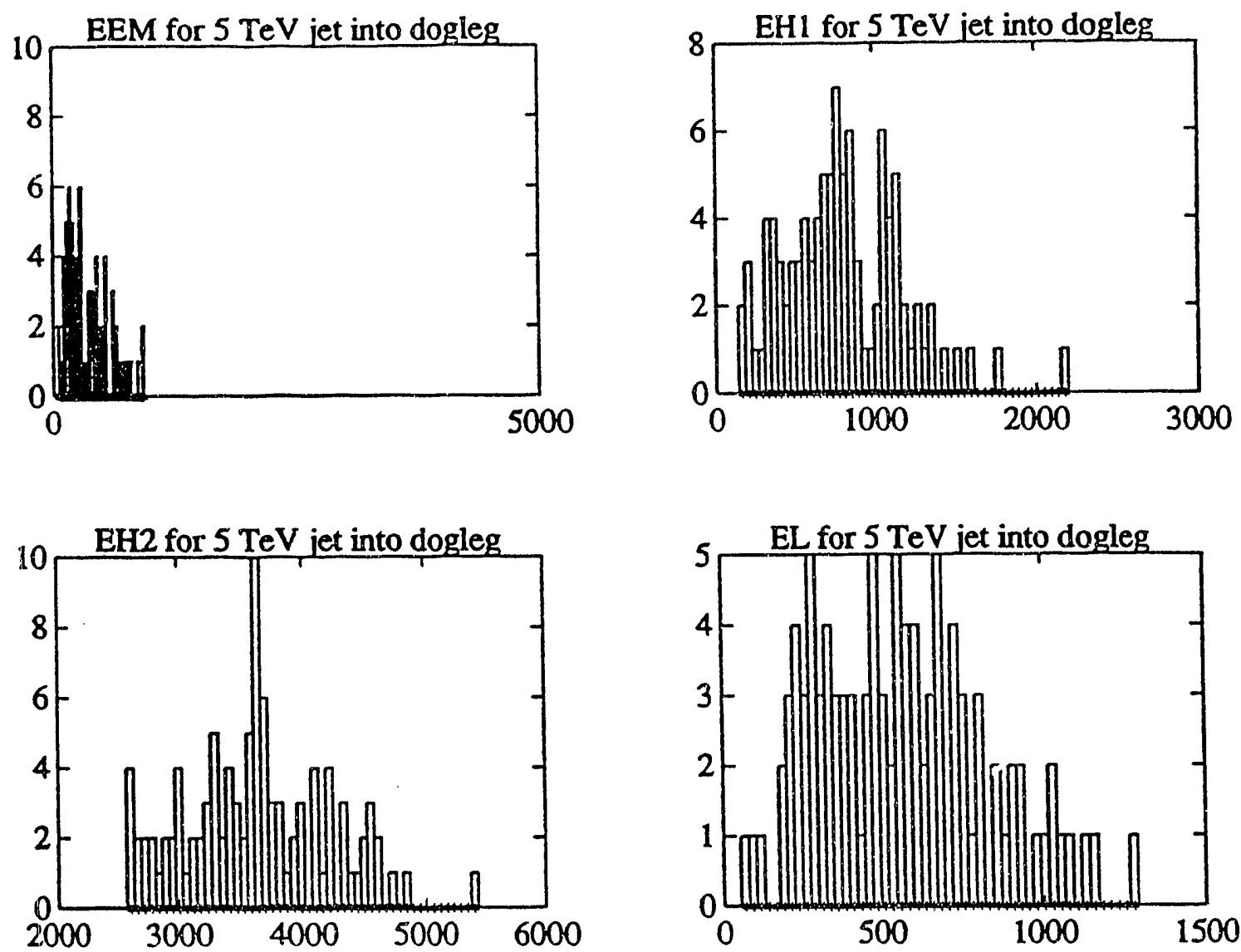

12. Energy deposition in EM, HAD1, HAD2, and EL for a $5 \mathrm{TeV}$ jet incident on the SDC calorimeter, at 90 degrees, directly down the center of a $2 \mathrm{~cm}$."crack" between adjacent wedges of EM and HAD1. 


\section{TEV JET INCIDENT \\ ON CALORIMETER}

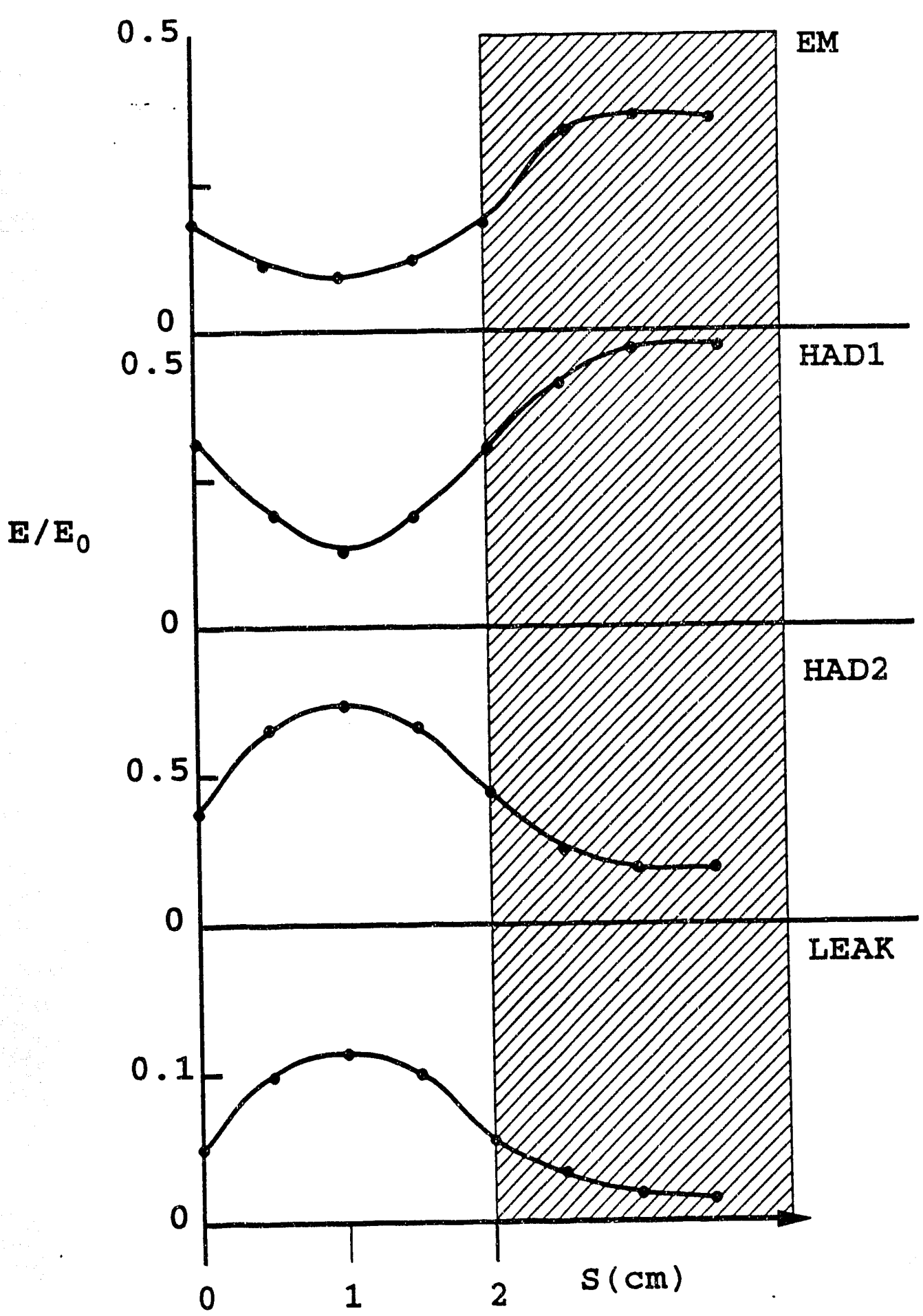

13. Fractional energy deposition in EM, HAD1, HAD2, and EL compartments of the SDC calorimeter as a function of distance of the jet axis from the assumed $2 \mathrm{~cm}$ crack between wedges of the SDC barrel. Clearly, the leakage energy rises from a value of $\sim 1 \%$ to a value of $\sim 10 \%$. The fluctuation in $\mathrm{EL}$ is conparable to the mean value of $\mathrm{EL}$. 

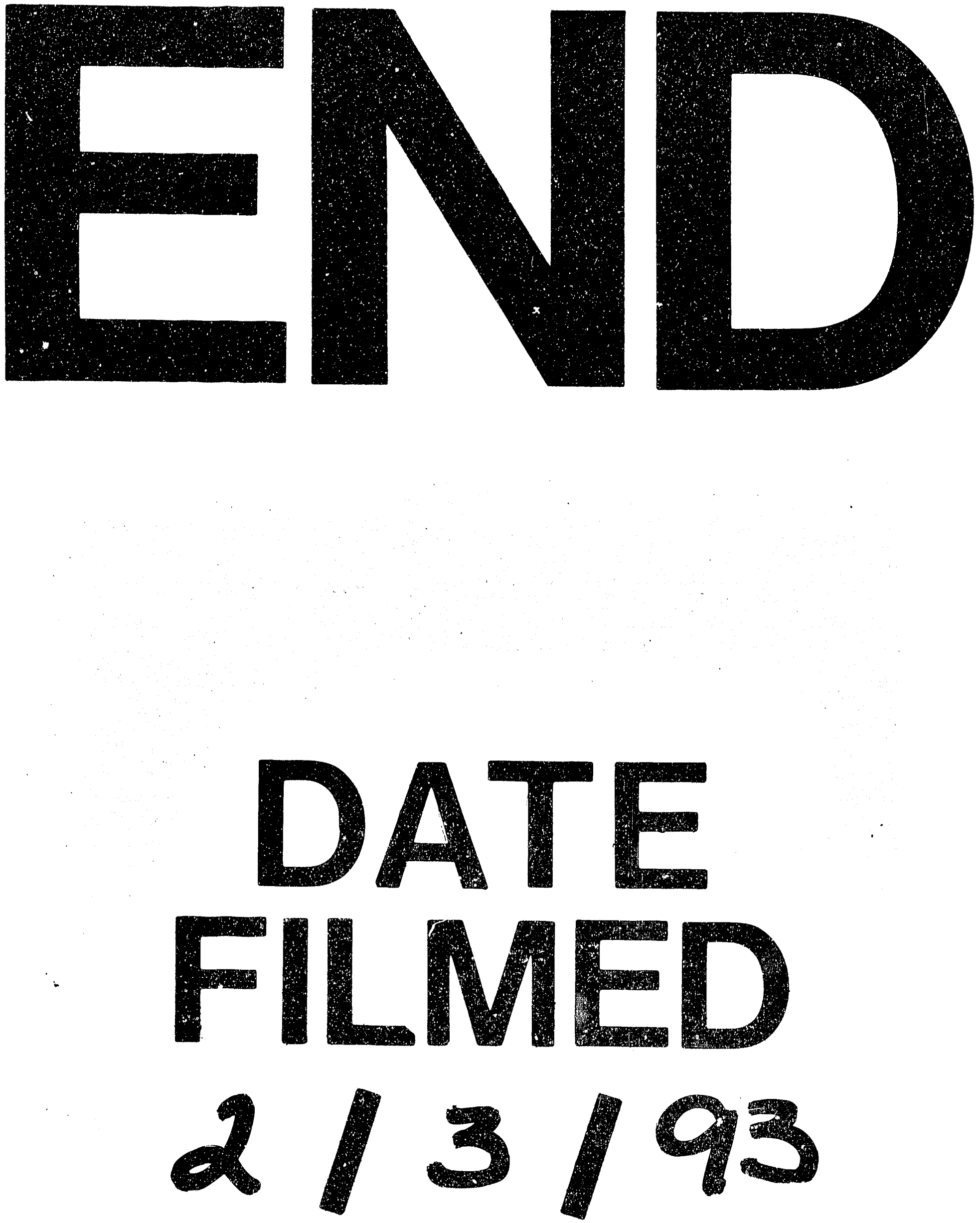
